\title{
Fixed point approximation of multivalued $\rho$-quasi-nonexpansive mappings in modular function spaces
}

\author{
Safeer Hussain Khan ${ }^{a}$, Mujahid Abbas ${ }^{b, c, *}$, Sartaj Alid \\ ${ }^{a}$ Department of Mathematics, Statistics and Physics, Qatar University, Doha 2713, Qatar. \\ ${ }^{b}$ Department of Mathematics, GC University, Katchery Road, Lahore 54000 Pakistan. \\ ${ }^{c}$ Department of Mathematics, King Abdulaziz University, P. O. Box 80203, Jeddah 21589, Saudi Arabia. \\ ${ }^{d}$ Department of Mathematics, Women University of Azad Jammu and Kashmir, Bagh, Azad Jammu and Kashmir, Pakistan. \\ Communicated by E. Savas
}

\begin{abstract}
The purpose of this paper is to investigate some convergence theorems in fixed point theory for $\rho$-quasi-nonexpansive multivalued mappings in modular function spaces using a faster iterative process. Examples are provided to validate our results. (C)2017 All rights reserved.
\end{abstract}

Keywords: Fixed point, multivalued $\rho$-quasi-nonexpansive mapping, iterative process, modular function space. 2010 MSC: 47H09, 47H10, 54C60.

\section{Introduction and preliminaries}

The fixed point theory in modular function spaces has recently received a good attention of researchers, see for example Khamsi and Kozolowski [5] and the references therein. Dhompongsa et al. [4] have proved that every $\rho$-contraction $T: C \rightarrow F_{\rho}(C)$ has a fixed point where $\rho$ is a convex function modular satisfying the so-called $\Delta_{2}$-type condition, $C$ is a nonempty $\rho$-bounded $\rho$-closed subset of $L_{\rho}$ and $F_{\rho}(C)$ a family of $\rho$-closed subsets of $C$. By using this result, they asserted the existence of fixed points for multivalued $\rho$-nonexpansive mappings. Their results are existential in nature. Dehaish and Kozlowski [3], for the first time, proved results on approximating fixed points in modular function spaces. However, these results are for single-valued mappings. Keeping in mind the importance and significance of multivalued mappings, Khan and Abbas [6], recently initiated the study of approximating fixed points of such mappings in modular function spaces. They approximated fixed points by convergence of a Mann iterative process (a one-step process) applied on multivalued $\rho$-nonexpansive mappings in modular function spaces. Khan et al. [7] constructed a three-step iterative process for multivalued mappings in Banach

\footnotetext{
*Corresponding author

Email addresses: saf eer@qu.edu.qa; safeerhussain5@yahoo.com (Safeer Hussain Khan), abbas.mujahid@gmail.com (Mujahid Abbas), sartajali2004@yahoo.com (Sartaj Ali)

doi:10.22436/jnsa.010.06.30
} 
spaces. This process is independent of both Mann and Ishikawa iterative processes in the sense that neither reduce to the other. Moreover, it is faster than all of Picard, Mann and Ishikawa iterative processes in case of contractions [1]. In this paper, as an attempt to have a significant advancement in the work of Khan and Abbas [6], we approximate fixed points of $\rho$-quasi-nonexpansive multivalued mappings in modular function spaces using a three step iterative process. This will generalize, unify, and improve many results in the contemporary literature.

Some basic facts and notations needed in this paper are recalled as follows.

Let $\Omega$ be a nonempty set and $\Sigma$ a nontrivial $\sigma$-algebra of subsets of $\Omega$. Let $\mathcal{P}$ be a $\delta$-ring of subsets of $\Omega$, such that $E \cap A \in \mathcal{P}$ for any $E \in \mathcal{P}$ and $A \in \Sigma$. Let us assume that there exists an increasing sequence of sets $K_{n} \in \mathcal{P}$ such that $\Omega=\cup K_{n}$ (for instance, $\mathcal{P}$ can be the class of sets of finite measure in a $\sigma$-finite measure space). By $1_{A}$, we denote the characteristic function of the set $A$ in $\Omega$. By $\mathcal{E}$ we denote the linear space of all simple functions with supports from $\mathcal{P}$. By $\mathcal{M}_{\infty}$ we will denote the space of all extended measurable functions, i.e., all functions $f: \Omega \rightarrow[-\infty, \infty]$ such that there exists a sequence $\left\{g_{n}\right\} \subset \mathcal{E}$, $\left|g_{n}\right| \leqslant|f|$ and $g_{n}(\omega) \rightarrow f(\omega)$ for all $\omega \in \Omega$.

Definition 1.1. Let $\rho: \mathcal{M}_{\infty} \rightarrow[0, \infty]$ be a nontrivial, convex, and even function. We say that $\rho$ is a regular convex function pseudomodular if

1. $\rho(0)=0$;

2. $\rho$ is monotone, i.e., $|f(\omega)| \leqslant|g(\omega)|$ for any $\omega \in \Omega$ implies $\rho(f) \leqslant \rho(g)$, where $f, g \in \mathcal{M}_{\infty}$;

3. $\rho$ is orthogonally subadditive, i.e., $\rho\left(f 1_{A \cup B}\right) \leqslant \rho\left(f 1_{A}\right)+\rho\left(f 1_{B}\right)$ for any $A, B \in \Sigma$ such that $A \cap B \neq \phi$, $f \in \mathcal{M}_{\infty}$;

4. $\rho$ has Fatou property, i.e., $\left|f_{n}(\omega)\right| \uparrow|f(\omega)|$ for all $\omega \in \Omega$ implies $\rho\left(f_{n}\right) \uparrow \rho(f)$, where $f \in \mathcal{M}_{\infty}$;

5. $\rho$ is order continuous in $\mathcal{E}$, i.e., $g_{n} \in \mathcal{E}$, and $\left|g_{n}(\omega)\right| \downarrow 0$ implies $\rho\left(g_{n}\right) \downarrow 0$.

A set $A \in \Sigma$ is said to be $\rho$-null if $\rho\left(g 1_{A}\right)=0$ for every $g \in \mathcal{E}$. A property $p(\omega)$ is said to hold $\rho$-almost everywhere ( $\rho$-a.e.) if the set $\{\omega \in \Omega: p(\omega)$ does not hold $\}$ is $\rho$-null. As usual, we identify any pair of measurable sets whose symmetric difference is $\rho$-null as well as any pair of measurable functions differing only on a $\rho$-null set. With this in mind, we define

$$
\mathcal{M}(\Omega, \Sigma, \mathcal{P}, \rho)=\left\{f \in \mathcal{M}_{\infty}:|f(\omega)|<\infty \rho \text {-a.e. }\right\},
$$

where $f \in \mathcal{M}(\Omega, \Sigma, \mathcal{P}, \rho)$ is actually an equivalence class of functions equal $\rho$-a.e. rather than an individual function. Where no confusion exists, we will write $\mathcal{M}$ instead of $\mathcal{M}(\Omega, \Sigma, \mathcal{P}, \rho)$.

Definition 1.2. Let $\rho$ be a regular function pseudomodular. We say that $\rho$ is a regular convex function modular if $\rho(f)=0$ implies $f=0$-a.e..

Definition 1.3. The convex function modular $\rho$ defines the modular function space $L_{\rho}$ as

$$
\mathrm{L}_{\rho}=\{f \in \mathcal{M}: \rho(\lambda f) \rightarrow 0 \text { as } \lambda \rightarrow 0\} .
$$

The class of all nonzero regular convex function modulars defined on $\Omega$ is denoted by $\mathfrak{R}$. Generally, the modular $\rho$ is not sub-additive and therefore does not behave as a norm or a distance. However, the modular space $\mathrm{L}_{\rho}$ can be equipped with an F-norm defined by

$$
\|f\|_{\rho}=\inf \left\{\alpha>0: \rho\left(\frac{f}{\alpha}\right) \leqslant \alpha\right\}
$$

In case $\rho$ is convex modular,

$$
\|f\|_{\rho}=\inf \left\{\alpha>0: \rho\left(\frac{f}{\alpha}\right) \leqslant 1\right\}
$$

defines a norm on the modular space $\mathrm{L}_{\rho}$, and is called the Luxemburg norm. 
Definition 1.4. Define $L_{\rho}^{0}=\left\{f \in L_{\rho}: \rho(f,\right.$.$\left.) is order continuous \right\}$ and the linear space $E_{\rho}=\left\{f \in L_{\rho}: \lambda f \in L_{\rho}^{0}\right.$ for every $\lambda>0\}$.

Definition 1.5. A nonzero regular convex function modular $\rho$ is said to satisfy the $\Delta_{2}$-condition, if $\sup _{n \geqslant 1} \rho\left(2 f_{n}, D_{k}\right) \rightarrow 0$ as $k \rightarrow \infty$ whenever $\left\{D_{k}\right\}$ decreases to $\phi$ and $\sup _{n \geqslant 1} \rho\left(f_{n}, D_{k}\right) \rightarrow 0$ as $k \rightarrow \infty$.

If $\rho$ is convex and satisfies the $\Delta_{2}$-condition, then $\mathrm{L}_{\rho}=\mathrm{E}_{\rho}$.

The following uniform convexity type properties of $\rho$ can be found in [5].

Definition 1.6. Let $\rho$ be a nonzero regular convex function modular defined on $\Omega$.

(i) Let $r>0, \varepsilon>0$. Define

$$
D_{1}(r, \epsilon)=\left\{(f, g): f, g \in L_{\rho}, \rho(f) \leqslant r, \rho(g) \leqslant r, \rho(f-g) \geqslant \varepsilon r\right\} .
$$

Let

$$
\delta_{1}(r, \epsilon)=\inf \left\{1-\frac{1}{r} \rho\left(\frac{f+g}{2}\right):(f, g) \in D_{1}(r, \epsilon)\right\} \text { if } D_{1}(r, \epsilon) \neq \phi,
$$

and $\delta_{1}(r, \epsilon)=1$ if $D_{1}(r, \epsilon)=\phi$. We say that $\rho$ satisfies (UC1) if for every $r>0, \epsilon>0, \delta_{1}(r, \epsilon)>0$. Note that for every $r>0, D_{1}(r, \epsilon) \neq \phi$ for $\epsilon>0$ small enough.

(ii) We say that $\rho$ satisfies (UUC1) if for every $s \geqslant 0, \epsilon>0$, there exists $\eta_{1}(s, \epsilon)>0$ depending only upon $s$ and $\epsilon$ such that $\delta_{1}(r, \epsilon)>\eta_{1}(s, \epsilon)>0$ for any $r>s$.

Note that (UC1) implies (UUC1).

Definition 1.7. Let $L_{\rho}$ be a modular space. The sequence $\left\{f_{n}\right\} \subset L_{\rho}$ is called:

- $\rho$-convergent to $f \in L_{\rho}$ if $\rho\left(f_{n}-f\right) \rightarrow 0$ as $n \rightarrow \infty$;

- $\rho$-Cauchy, if $\rho\left(f_{n}-f_{m}\right) \rightarrow 0$ as $n$ and $m \rightarrow \infty$.

Note that $\rho$-convergence does not imply $\rho$-Cauchy since $\rho$ does not satisfy the triangle inequality. In fact, one can show that this will happen if and only if $\rho$ satisfies the $\Delta_{2}$-condition.

The $\rho$-distance from an $f \in L_{\rho}$ to a set $\mathrm{D} \subset \mathrm{L}_{\rho}$ is given as follows:

$$
\operatorname{dist}_{\rho}(f, D)=\inf \{\rho(f-h): h \in D\} .
$$

Definition 1.8. A subset $\mathrm{D} \subset \mathrm{L}_{\rho}$ is called

- $\rho$-closed if the $\rho$-limit of a $\rho$-convergent sequence of D always belongs to D;

- $\rho$-a.e. closed if the $\rho$-a.e. limit of a $\rho$-a.e. convergent sequence of $D$ always belongs to $D$;

- $\rho$-compact if every sequence in $D$ has a $\rho$-convergent subsequence in $D$;

- $\rho$-a.e. compact if every sequence in $\mathrm{D}$ has a $\rho$-a.e. convergent subsequence in $\mathrm{D}$;

- $\rho$-bounded if

$$
\operatorname{diam}_{\rho}(D)=\sup \{\rho(f-g): f, g \in D\}<\infty .
$$

A set $D \subset L_{\rho}$ is called $\rho$-proximinal if for each $f \in L_{\rho}$ there exists an element $g \in D$ such that $\rho(f-g)=$ $\operatorname{dist}_{\rho}(f, D)$. We shall denote the family of nonempty $\rho$-bounded $\rho$-proximinal subsets of $D$ by $P_{\rho}(D)$, the family of nonempty $\rho$-closed $\rho$-bounded subsets of $D$ by $C_{\rho}(D)$ and the family of $\rho$-compact subsets of $D$ by $K_{\rho}(D)$. Let $H_{\rho}(\cdot, \cdot)$ be the $\rho$-Hausdorff distance on $C_{\rho}\left(L_{\rho}\right)$, that is,

$$
H_{\rho}(A, B)=\max \left\{\sup _{f \in A} \operatorname{dist}_{\rho}(f, B), \sup _{g \in B} \operatorname{dist}_{\rho}(g, A)\right\}, A, B \in C_{\rho}\left(L_{\rho}\right) .
$$

A multivalued mapping $\mathrm{T}: \mathrm{D} \rightarrow \mathrm{C}_{\rho}\left(\mathrm{X}_{\rho}\right)$ is said to be: 
(a) $\rho$-nonexpansive if

$$
H_{\rho}(T f, T g) \leqslant \rho(f-g) \text { for all } f, g \in D .
$$

(b) $\rho$-quasi-nonexpansive mapping if $H_{\rho}(T f, p) \leqslant \rho(f-p)$ for all $f \in D$ and $p \in F_{\rho}(T)$.

Definition 1.9. Let $\rho \in \mathfrak{R}$. The growth function $\omega_{\rho}$ of a function modular $\rho$ is defined as:

$$
\omega_{\rho}(t)=\sup \left\{\frac{\rho(t f)}{\rho(f)}, 0 \leqslant \rho(f)<\infty\right\} \text { for all } 0 \leqslant t<\infty .
$$

Observe that $\omega_{\rho}(t) \leqslant 1$ for all $t \in[0,1]$. Some properties of the growth function $\omega$ can be found in [5].

Having the fact in mind that triangle inequality is not available in modular function spaces, the following theorem is very useful. It can be found in [2].

Theorem 1.10. Let $\rho \in \mathfrak{R}$ satisfy the $\Delta_{2}$-condition. Let $\left\{\mathrm{f}_{n}\right\}$ and $\left\{\mathrm{g}_{\mathrm{n}}\right\}$ be two sequences in $\mathrm{L}_{\rho}$. Then

$$
\lim _{n \rightarrow \infty} \rho\left(g_{n}\right)=0 \text { implies } \limsup _{n \rightarrow \infty} \rho\left(f_{n}+g_{n}\right)=\limsup _{n \rightarrow \infty} \rho\left(f_{n}\right)
$$

and

$$
\lim _{n \rightarrow \infty} \rho\left(g_{n}\right)=0 \text { implies } \liminf _{n \rightarrow \infty} \rho\left(f_{n}+g_{n}\right)=\liminf _{n \rightarrow \infty} \rho\left(f_{n}\right) .
$$

A sequence $\left\{t_{n}\right\} \subset(0,1)$ is called bounded away from 0 if there exists $a>0$ such that $t_{n} \geqslant a$ for every $n \in \mathbb{N}$. Similarly, $\left\{t_{n}\right\} \subset(0,1)$ is called bounded away from 1 if there exists $b<1$ such that $t_{n} \leqslant b$ for every $n \in \mathbb{N}$.

The following lemma can be seen as an analogue of a famous lemma due to Schu in Banach spaces. It can be seen in [5].

Lemma 1.11. Let $\rho \in \Re$ satisfy (UUC1) and let $\left\{t_{k}\right\} \subset(0,1)$ be bounded away from 0 and 1 . If there exists $R>0$ such that

$$
\limsup _{n \rightarrow \infty} \rho\left(f_{n}\right) \leqslant R, \limsup _{n \rightarrow \infty} \rho\left(g_{n}\right) \leqslant R \text {, and } \lim _{n \rightarrow \infty} \rho\left(t_{n} f_{n}+\left(1-t_{n}\right) g_{n}\right)=R \text {, }
$$

then

$$
\lim _{n \rightarrow \infty} \rho\left(f_{n}-g_{n}\right)=0 .
$$

Definition 1.12. A function $f \in L_{\rho}$ is called a fixed point of $T: L_{\rho} \rightarrow P_{\rho}(D)$ if $f \in T f$. The set of all fixed points of $T$ will be denoted by $F_{\rho}(T)$.

The proof of the following lemma can be found in Khan and Abbas [6].

Lemma 1.13. Let $\mathrm{T}: \mathrm{D} \rightarrow \mathrm{P}_{\rho}(\mathrm{D})$ be a multivalued mapping and

$$
P_{\rho}^{\top}(f)=\left\{g \in T f: \rho(f-g)=\operatorname{dist}_{\rho}(f, T f)\right\} .
$$

Then the following are equivalent

(1) $f \in F_{\rho}(T)$, that is, $f \in T f$.

(2) $\mathrm{P}_{\rho}^{\mathrm{T}}(\mathrm{f})=\{\mathrm{f}\}$, that is, $\mathrm{f}=\mathrm{g}$ for each $\mathrm{g} \in \mathrm{P}_{\rho}^{\mathrm{T}}(\mathrm{f})$.

(3) $f \in F\left(P_{\rho}^{\top}(f)\right)$, that is, $f \in P_{\rho}^{\top}(f)$. Further $F_{\rho}(T)=F\left(P_{\rho}^{\top}(f)\right)$ where $F\left(P_{\rho}^{\top}(f)\right)$ denotes the set of fixed points of $P_{\rho}^{\top}(f)$.

The following definition can also be found in [6].

Definition 1.14. A multivalued mapping $T: D \rightarrow P_{\rho}(D)$ is said to satisfy condition (I) if there exists a nondecreasing function $l:[0, \infty) \rightarrow[0, \infty)$ with $l(0)=0, l(r)>0$ for all $r \in(0, \infty)$ such that $\operatorname{dist}_{\rho}(f, T f) \geqslant$ $l\left(\operatorname{dist}_{\rho}\left(f, F_{\rho}(T)\right)\right)$ for all $f \in D$. 
We give the following example to show that a mapping $\mathrm{T}$ indeed satisfies the condition (I).

Example 1.15. Let the set of real numbers $\mathbb{R}$ be the space modulared as $\rho(f)=|f|^{2}$. Let $D=\left\{f \in L_{\rho}: 0 \leqslant f\right.$ $\leqslant 2\}$, define $\mathrm{T}: \mathrm{D} \rightarrow \mathrm{P}_{\rho}(\mathrm{D})$ as:

$$
\operatorname{Tf}=\left[0, \frac{f+1}{2}\right] .
$$

Obviously $D$ is a $\rho$-compact subset of $\mathbb{R}$. Note that $F_{\rho}(T)=[0,1]$. Let $\alpha=\beta=\gamma=\frac{1}{2}$. Define a continuous nondecreasing function $l:[0, \infty) \rightarrow[0, \infty)$ by $l(r)=\frac{r}{4}$. Note that $\operatorname{dist}_{\rho}(f, T f) \geqslant l\left(\operatorname{dist}_{\rho}\left(f, F_{\rho}(T)\right)\right)$ for all $f \in D$. Indeed, if $f \in F_{\rho}(T)=[0,1]$, then obviously

$$
\operatorname{dist}_{\rho}(f, T f)=0=l\left(\operatorname{dist}_{\rho}\left(f, F_{\rho}(T)\right)\right) .
$$

If $f \in(2, \infty)$, then

$$
\operatorname{dist}_{\rho}\left(f,\left[0, \frac{f+1}{2}\right]\right)=\left|f-\left(\frac{f+1}{2}\right)\right|=\frac{f-1}{2}
$$

and

$$
l\left(\operatorname{dist}_{\rho}\left(f, F_{\rho}(T)\right)\right)=l\left(\operatorname{dist}_{\rho}(f,[0,1])\right)=l(|f-1|)=\frac{f-1}{4}
$$

so $\operatorname{dist}_{\rho}(f, T f) \geqslant l\left(\operatorname{dist}_{\rho}\left(f, F_{\rho}(T)\right)\right)$ for all $f \in D$.

\section{Approximating fixed points in modular function spaces}

In this section, we prove a very important result which plays a vital role in establishing our result for approximating fixed points of multivalued $\rho$-quasi-nonexpansive mappings in modular function spaces. We will be using a three step faster iterative process [7].

Recall that a multivalued mapping $\mathrm{T}: \mathrm{D} \rightarrow \mathrm{C}_{\rho}\left(\mathrm{L}_{\rho}\right)$ is called $\rho$-quasi-nonexpansive if

$$
H_{\rho}(T f, p) \leqslant \rho(f-p) \text { for all } f \in D \text { and } p \in F_{\rho}(T) .
$$

Theorem 2.1. Let $\rho \in \mathfrak{R}$ satisfy (UUC1) and $\Delta_{2}$-condition. Let $\mathrm{D}$ a nonempty $\rho$-closed, $\rho$-bounded, and convex subset of $\mathrm{L}_{\rho}$. Let $\mathrm{T}: \mathrm{D} \rightarrow \mathrm{P}_{\rho}(\mathrm{D})$ be a multivalued mapping such that $\mathrm{P}_{\rho}^{\mathrm{T}}$ is a $\rho$-quasi-nonexpansive mapping. Suppose that $\mathrm{F}_{\rho}(\mathrm{T}) \neq \phi$. Let $\left\{\mathrm{f}_{\mathrm{n}}\right\} \subset \mathrm{D}$ be defined by three step iterative process as:

$$
f_{n+1}=(1-\alpha) v_{n}+\alpha w_{n} g_{n}=(1-\beta) u_{n}+\beta w_{n} h_{n}=(1-\gamma) f_{n}+\gamma u_{n},
$$

where $u_{n} \in P_{\rho}^{\top}\left(f_{n}\right), v_{n} \in P_{\rho}^{\top}\left(g_{n}\right), w_{n} \in P_{\rho}^{\top}\left(h_{n}\right)$, and $0<\alpha, \beta, \gamma<1$. Then $\lim _{n \rightarrow \infty} \rho\left(f_{n}-p\right)$ exists for all $p \in F_{\rho}(T)$, and $\lim _{n \rightarrow \infty} \operatorname{dist}_{\rho}\left(f_{n}, P_{\rho}^{\top}\left(f_{n}\right)\right)=0$.

Proof. Let $p \in F_{\rho}(T)$. By Lemma 1.13, $\mathrm{P}_{\rho}^{\top}(p)=\{p\}$, and $F_{\rho}(T)=F\left(P_{\rho}^{\top}\right)$. Now

$$
\rho\left(f_{n+1}-p\right)=\rho\left[(1-\alpha) v_{n}+\alpha w_{n}-p\right]=\rho\left[(1-\alpha)\left(v_{n}-p\right)+\alpha\left(w_{n}-p\right)\right] .
$$

The convexity of $\rho$ implies

$$
\begin{aligned}
\rho\left(f_{n+1}-p\right) \leqslant(1-\alpha) \rho\left(v_{n}-p\right)+\alpha \rho\left(w_{n}-p\right) & \leqslant(1-\alpha) H_{\rho}\left(P_{\rho}^{\top}\left(g_{n}\right), P_{\rho}^{\top}(p)\right)+\alpha H_{\rho}\left(P_{\rho}^{\top}\left(h_{n}\right), P_{\rho}^{\top}(p)\right) \\
& \leqslant(1-\alpha) \rho\left(g_{n}-p\right)+\alpha \rho\left(h_{n}-p\right) .
\end{aligned}
$$

Now

$$
\left.\rho\left(g_{n}-p\right)=\rho\left[(1-\beta) u_{n}+\beta w_{n}\right)-p\right]=\rho\left[(1-\beta)\left(u_{n}-p\right)+\beta\left(w_{n}-p\right)\right] .
$$

Again by convexity of $\rho$, we have

$$
\rho\left(g_{n}-p\right) \leqslant(1-\beta) \rho\left(u_{n}-p\right)+\beta \rho\left(w_{n}-p\right)
$$




$$
\begin{aligned}
& \leqslant(1-\beta) H_{\rho}\left(P_{\rho}^{\top}\left(f_{n}\right), P_{\rho}^{\top}(p)\right)+\beta H_{\rho}\left(P_{\rho}^{\top}\left(h_{n}\right), P_{\rho}^{\top}(p)\right) \\
& \leqslant(1-\beta) \rho\left(f_{n}-p\right)+\beta \rho\left(h_{n}-p\right) .
\end{aligned}
$$

Next,

$$
\left.\rho\left(h_{n}-p\right)=\rho\left[(1-\gamma) f_{n}+\gamma u_{n}-p\right)\right] .
$$

Using convexity of $\rho$ once again, we get

$$
\begin{aligned}
\left.\rho\left(h_{n}-p\right)=\rho\left[(1-\gamma) f_{n}+\gamma u_{n}-p\right)\right] & \leqslant(1-\gamma) \rho\left(f_{n}-p\right)+\gamma \rho\left(u_{n}-p\right) \\
& \leqslant(1-\gamma) \rho\left(f_{n}-p\right)+\gamma H_{\rho}\left(P_{\rho}^{\top}\left(f_{n}\right), P_{\rho}^{\top}(p)\right) \\
& \leqslant(1-\gamma) \rho\left(f_{n}-p\right)+\gamma \rho\left(f_{n}-p\right)=\rho\left(f_{n}-p\right) .
\end{aligned}
$$

That is,

$$
\rho\left(h_{n}-p\right) \leqslant \rho\left(f_{n}-p\right) .
$$

Thus

$$
\rho\left(g_{n}-p\right) \leqslant(1-\beta) \rho\left(f_{n}-p\right)+\beta \rho\left(h_{n}-p\right) \leqslant(1-\beta) \rho\left(f_{n}-p\right)+\beta \rho\left(f_{n}-p\right)=\rho\left(f_{n}-p\right)
$$

implies

$$
\rho\left(g_{n}-p\right) \leqslant \rho\left(f_{n}-p\right)
$$

Hence

$$
\rho\left(f_{n+1}-p\right) \leqslant(1-\alpha) \rho\left(g_{n}-p\right)+\alpha \rho\left(h_{n}-p\right) \leqslant(1-\alpha) \rho\left(f_{n}-p\right)+\alpha \rho\left(f_{n}-p\right)=\rho\left(f_{n}-p\right) .
$$

This shows that $\lim _{n \rightarrow \infty} \rho\left(f_{n}-p\right)$ exists for each $p \in F_{\rho}(T)$. Suppose that

$$
\lim _{n \rightarrow \infty} \rho\left(f_{n}-p\right)=L,
$$

where $L \geqslant 0$. We now prove that $\lim _{n \rightarrow \infty} \operatorname{dist}_{\rho}\left(f_{n}, P_{\rho}^{\top}\left(f_{n}\right)\right)=0$. In view of

$$
\operatorname{dist}_{\rho}\left(f_{n}, P_{\rho}^{\top}\left(f_{n}\right)\right) \leqslant \rho\left(f_{n}-u_{n}\right),
$$

it suffices to prove that

$$
\lim _{n \rightarrow \infty} \rho\left(f_{n}-u_{n}\right)=0
$$

Now

$$
\rho\left(u_{n}-p\right) \leqslant H_{\rho}\left(P_{\rho}^{\top}\left(f_{n}\right), P_{\rho}^{\top}(p)\right) \leqslant \rho\left(f_{n}-p\right)
$$

implies

$$
\limsup _{n \rightarrow \infty} \rho\left(u_{n}-p\right) \leqslant \limsup _{n \rightarrow \infty} \rho\left(f_{n}-p\right)
$$

and so by (2.4) we have

$$
\limsup _{n \rightarrow \infty} \rho\left(u_{n}-p\right) \leqslant L
$$

Also from (2.2), we have

$$
\limsup _{n \rightarrow \infty} \rho\left(h_{n}-p\right) \leqslant \limsup _{n \rightarrow \infty} \rho\left(f_{n}-p\right),
$$

so that

$$
\limsup _{n \rightarrow \infty} \rho\left(h_{n}-p\right) \leqslant L .
$$

Similarly, from (2.3) and (2.4)

$$
\limsup _{n \rightarrow \infty} \rho\left(g_{n}-p\right) \leqslant L .
$$


Moving forward, the inequality

$$
\rho\left(v_{n}-p\right) \leqslant H_{\rho}\left(P_{\rho}^{\top}\left(g_{n}\right), P_{\rho}^{\top}(p)\right) \leqslant \rho\left(g_{n}-p\right) \leqslant \rho\left(f_{n}-p\right)
$$

yields

$$
\limsup _{n \rightarrow \infty} \rho\left(v_{n}-p\right) \leqslant \limsup _{n \rightarrow \infty} \rho\left(f_{n}-p\right),
$$

so that

$$
\limsup _{n \rightarrow \infty} \rho\left(v_{n}-p\right) \leqslant L
$$

Similarly,

$$
\limsup _{n \rightarrow \infty} \rho\left(w_{n}-p\right) \leqslant L
$$

As

$$
\left.\lim _{n \rightarrow \infty} \rho\left(f_{n+1}-p\right)=\lim _{n \rightarrow \infty} \rho\left[(1-\alpha) v_{n}+\alpha w_{n}\right)-p\right]=\lim _{n \rightarrow \infty} \rho\left[\left(1-\alpha_{n}\right)\left(v_{n}-p\right)+\alpha_{n}\left(w_{n}-p\right)\right]=L,
$$

from (2.7), (2.8), (2.9), and Lemma 1.11, we have

$$
\lim _{n \rightarrow \infty} \rho\left(v_{n}-w_{n}\right)=0 .
$$

Fix $\varepsilon>0$. There exists $n_{0} \in \mathbb{N}$ such that $\rho\left(v_{n}-w_{n}\right)<\varepsilon$ for all $n \geqslant n_{0}$. Note that $\rho\left(\alpha\left(v_{n}-w_{n}\right)\right) \leqslant$ $\omega_{\rho}(\alpha) \rho\left(v_{n}-w_{n}\right)<\rho\left(v_{n}-w_{n}\right)<\varepsilon$ and hence

$$
\lim _{n \rightarrow \infty} \rho\left(\alpha\left(v_{n}-w_{n}\right)\right)=0
$$

Again

$$
\left.\rho\left(f_{n+1}-p\right)=\rho\left[(1-\alpha) v_{n}+\alpha w_{n}\right)-p\right]=\rho\left[\left(v_{n}-p\right)+\alpha\left(w_{n}-v_{n}\right)\right] .
$$

By using Theorem 1.10, we have

$$
\liminf _{n \rightarrow \infty} \rho\left[\left(v_{n}-p\right)+\alpha\left(w_{n}-v_{n}\right)\right]=\lim _{n \rightarrow \infty} \inf \rho\left(v_{n}-p\right) .
$$

That is,

$$
L=\lim _{n \rightarrow \infty} \inf \rho\left(v_{n}-p\right)
$$

From (2.7) and (2.11), we have

$$
\lim _{n \rightarrow \infty} \rho\left(v_{n}-p\right)=L .
$$

Again by using (2.10) and Theorem 1.10, we have

$$
L=\liminf _{n \rightarrow \infty} \rho\left(v_{n}-p\right)=\liminf _{n \rightarrow \infty} \rho\left[\left(v_{n}-w_{n}\right)+\left(w_{n}-p\right)\right]=\liminf _{n \rightarrow \infty} \rho\left(w_{n}-p\right) .
$$

But

Therefore

$$
\rho\left(w_{n}-p\right) \leqslant H_{\rho}\left(P_{\rho}^{\top}\left(h_{n}\right), P_{\rho}^{\top}(p)\right) \leqslant \rho\left(h_{n}-p\right) .
$$

$$
L \leqslant \lim _{n \rightarrow \infty} \inf \rho\left(h_{n}-p\right)
$$

From (2.6) and (2.12), we have

$$
\lim _{n \rightarrow \infty} \rho\left(h_{n}-p\right)=L
$$

That is,

$$
\lim _{n \rightarrow \infty} \rho\left[(1-\gamma)\left(f_{n}-p\right)+\gamma\left(u_{n}-p\right)\right]=L .
$$

Thus from (2.4), (2.5), and Lemma 1.11, we have

$$
\lim _{n \rightarrow \infty} \rho\left(f_{n}-u_{n}\right)=0 .
$$

Hence $\lim _{n \rightarrow \infty} \operatorname{dist}_{\rho}\left(f_{n}, P_{\rho}^{\top}\left(f_{n}\right)\right)=0$. 
Now we are going to present our convergence result for approximating fixed points of multivalued $\rho$-quasi-nonexpansive mappings in modular function spaces using three step iterative process (2.1) as follows.

Theorem 2.2. Let $\rho \in \mathfrak{R}$ satisfy (UUC1) and $\Delta_{2}$-condition. Let $\mathrm{D}$ a nonempty $\rho$-closed, $\rho$-bounded, and convex subset of $\mathrm{L}_{\rho}$. Let $\mathrm{T}: \mathrm{D} \rightarrow \mathrm{P}_{\rho}(\mathrm{D})$ be a multivalued mapping such that $\mathrm{P}_{\rho}^{\mathrm{T}}$ is $\rho$-quasi-nonexpansive mapping. Suppose that $\mathrm{F}_{\rho}(T) \neq \phi$. Let $\left\{\mathrm{f}_{\mathrm{n}}\right\}$ be defined as in Theorem 2.1. Then $\left\{\mathrm{f}_{n}\right\}$-converges to a fixed point of $\mathrm{T}$.

Proof. From $\rho$-compactness of $D$, there exists a subsequence $\left\{f_{n_{k}}\right\}$ of $\left\{f_{n}\right\}$ such that $\lim _{k \rightarrow \infty}\left(f_{n_{k}}-q\right)=0$ for some $q \in D$. To prove that $q$ is a fixed point of $T$, let $g$ be an arbitrary point in $P_{\rho}^{\top}(q)$ and $f$ in $P_{\rho}^{\top}\left(f_{n_{k}}\right)$. Note that

$$
\begin{aligned}
\rho\left(\frac{q-g}{3}\right) & \leqslant \rho\left(\frac{q-f_{n_{k}}}{3}\right)+\rho\left(\frac{f_{n_{k}}-f}{3}\right)+\rho\left(\frac{f-g}{3}\right) \\
& \leqslant \frac{1}{3} \rho\left(q-f_{n_{k}}\right)+\frac{1}{3} \rho\left(f_{n_{k}}-f\right)+\frac{1}{3} \rho(f-g) \\
& \leqslant \rho\left(q-f_{n_{k}}\right)+\operatorname{dist}_{\rho}\left(f_{n_{k}}, P_{\rho}^{\top}\left(f_{n_{k}}\right)\right)+\operatorname{dist}_{\rho}\left(P_{\rho}^{\top}\left(f_{n_{k}}\right), g\right) \\
& \leqslant \rho\left(q-f_{n_{k}}\right)+\operatorname{dist}_{\rho}\left(f_{n_{k}}, P_{\rho}^{\top}\left(f_{n_{k}}\right)\right)+H_{\rho}\left(P_{\rho}^{\top}\left(f_{n_{k}}\right), P_{\rho}^{\top}(q)\right) \\
& \leqslant \rho\left(q-f_{n_{k}}\right)+\operatorname{dist}_{\rho}\left(f_{n_{k}}, P_{\rho}^{\top}\left(f_{n_{k}}\right)\right)+\rho\left(q-f_{n_{k}}\right) .
\end{aligned}
$$

This gives $\rho(q-g)=0$ using Theorem 2.1. Hence $q \in F\left(P_{\rho}^{T}\right)=F_{\rho}(T)$. That is, $\left\{f_{n}\right\} \rho$-converges to a fixed point of $\mathrm{T}$.

Theorem 2.3. Let $\rho \in \mathfrak{R}$ satisfy (UUC1) and $\Delta_{2}$-condition. Let $\mathrm{D}$ a nonempty $\rho$-closed, $\rho$-bounded, and convex subset of $\mathrm{L}_{\rho}$. Let $\mathrm{T}: \mathrm{D} \rightarrow \mathrm{P}_{\rho}(\mathrm{D})$ be a multivalued mapping with $\mathrm{F}_{\rho}(\mathrm{T}) \neq \phi$ and satisfying condition (I) such that $\mathrm{P}_{\rho}^{\mathrm{T}}$ is $\rho$-quasi-nonexpansive mapping. Let $\left\{\mathrm{f}_{\mathrm{n}}\right\}$ be defined as in Theorem 2.1. Then $\left\{\mathrm{f}_{n}\right\} \rho$-converges to a fixed point of $\mathrm{T}$.

Proof. From Theorem 2.1, $\lim _{n \rightarrow \infty} \rho\left(f_{n}-p\right)$ exists for all $p \in F\left(P_{\rho}^{T}\right)=F_{\rho}(T)$. If $\lim _{n \rightarrow \infty} \rho\left(f_{n}-p\right)=0$, there is nothing to prove. We assume $\lim _{n \rightarrow \infty} \rho\left(f_{n}-p\right)=L>0$. Now from Theorem 2.1, $\rho\left(f_{n+1}-p\right) \leqslant \rho\left(f_{n}-p\right)$ so that

$$
\operatorname{dist}_{\rho}\left(f_{n+1}, F_{\rho}(T)\right) \leqslant \operatorname{dist}_{\rho}\left(f_{n}, F_{\rho}(T)\right) .
$$

Hence $\lim _{n \rightarrow \infty} \operatorname{dist}_{\rho}\left(f_{n}, F_{\rho}(T)\right)$ exists. We show that $\lim _{n \rightarrow \infty} \operatorname{dist}_{\rho}\left(f_{n}, F_{\rho}(T)\right)=0$. By using condition (I) and Theorem 2.1, we have

$$
\lim _{n \rightarrow \infty} l\left(\operatorname{dist}_{\rho}\left(f_{n}, F_{\rho}(T)\right)\right) \leqslant \lim _{n \rightarrow \infty} \operatorname{dist}_{\rho}\left(f_{n}, T f_{n}\right)=0
$$

That is,

$$
\lim _{n \rightarrow \infty} l\left(\operatorname{dist}_{\rho}\left(f_{n}, F_{\rho}(T)\right)\right)=0 .
$$

Since $l$ is a nondecreasing function and $l(0)=0$, it follows that $\lim _{n \rightarrow \infty} \operatorname{dist}_{\rho}\left(f_{n}, F_{\rho}(T)\right)=0$.

Next, we show that $\left\{f_{n}\right\}$ is a $\rho$-Cauchy sequence in D. Let $\varepsilon>0$ be arbitrarily chosen. Since $\lim _{n \rightarrow \infty} \operatorname{dist}_{\rho}\left(f_{n}, F_{\rho}(T)\right)=0$, there exists a constant $n_{0}$ such that for all $n \geqslant n_{0}$, we have

$$
\operatorname{dist}_{\rho}\left(f_{n}, F_{\rho}(T)\right)<\frac{\varepsilon}{2} .
$$

In particular, $\inf \left\{\rho\left(f_{n_{0}}-p\right): p \in F_{\rho}(T)\right\}<\frac{\varepsilon}{2}$. There must exist a $p^{*} \in F_{\rho}(T)$ such that

$$
\rho\left(f_{n_{0}}-p^{*}\right)<\varepsilon .
$$

Now for $m, n \geqslant n_{0}$, we have

$$
\rho\left(\frac{f_{n+m}-f_{n}}{2}\right) \leqslant \frac{1}{2} \rho\left(f_{n+m}-p^{*}\right)+\frac{1}{2} \rho\left(f_{n}-p^{*}\right) \leqslant \rho\left(f_{n_{0}}-p^{*}\right)<\varepsilon .
$$

Hence $\left\{f_{n}\right\}$ is a $\rho$-Cauchy sequence in a $\rho$-closed subset $D$ of $L_{\rho}$, and so it must converge in D. Let 
$\lim _{n \rightarrow \infty} f_{n}=q$. Finally, $q \in F_{\rho}(T)$ now follows from Theorem 2.2.

Example 2.4 ([8]). Let $(X,\|\|$.$) be a normed space, then \|$.$\| is a modular. But the converse is not true.$

Example $2.5([8])$. Let $(X,\|\|$.$) be normed space. For any k \geqslant 1,\|\cdot\|^{k}$ is a modular on $X$.

In the following example, we verify our Theorem 2.2 .

Example 2.6. Let the real number system $\mathbb{R}$ be the space modulared as

$$
\rho(f)=|f|^{k}, k \geqslant 1 .
$$

Let $\mathrm{D}=\left\{\mathrm{f} \in \mathrm{L}_{\rho}: 0 \leqslant \mathrm{f} \leqslant 1\right\}$. Define $\mathrm{T}: \mathrm{D} \rightarrow \mathrm{P}_{\rho}(\mathrm{D})$ as:

$$
\mathrm{Tf}=\left[0, \frac{2 \mathrm{f}+1}{4}\right] \text {. }
$$

Obviously $\mathrm{D}$ is a nonempty $\rho$-compact, $\rho$-bounded, and convex subset of $\mathrm{L}_{\rho}=\mathbb{R}$ which satisfies (UC1) condition. As $\rho(f)=|f|^{k}, k \geqslant 1$ is homogeneous of degree $k$, so (UUC1) holds. Note that $F_{\rho}(T)=\left[0, \frac{1}{2}\right] \neq$ $\phi$. Let $\alpha=\beta=\gamma=\frac{1}{2}$. Observe that $\mathrm{P}_{\rho}^{\mathrm{T}}(\mathrm{f})=\{\mathrm{f}\}$ when $\mathrm{f} \in\left[0, \frac{1}{2}\right]$. In case $\mathrm{f} \notin\left[0, \frac{1}{2}\right]$,

$$
\begin{aligned}
P_{\rho}^{\top}(f) & =\left\{g \in T f: \rho(f-g)=\operatorname{dist}_{\rho}(f, T f)=\operatorname{dist}_{\rho}\left(f,\left[0, \frac{2 f+1}{4}\right]\right)\right\} \\
& =\left\{g \in T f:|f-g|^{k}=\operatorname{dist}_{\rho}\left(f,\left[0, \frac{2 f+1}{4}\right]\right)\right\} \\
& =\left\{g \in T f:|f-g|^{k}=\left|f-\frac{2 f+1}{4}\right|^{k}=\left|\frac{2 f-1}{4}\right|^{k}\right\} \\
& =\left\{g \in T f:|f-g|=\left|\frac{2 f-1}{4}\right|\right\} \\
& =\left\{g \in T f: f-g=\frac{2 f-1}{4}\right\} \text { because } f>g \text { for all } g \in \text { Tf where } f \in\left(\frac{1}{2}, 1\right] . \\
& \Rightarrow P_{\rho}^{\top}(f)=\left\{g=\frac{2 f+1}{4}\right\} .
\end{aligned}
$$

Next, we prove that $P_{\rho}^{\top}(f)$ is $\rho$-quasi-nonexpansive for all $f \in D$. The case of $f \in\left[0, \frac{1}{2}\right]$ is trivial. Thus, we take $f \in\left(\frac{1}{2}, 1\right]$.

$$
H_{\rho}\left(P_{\rho}^{\top}(f), P_{\rho}^{\top}(p)\right)=H_{\rho}\left(\frac{2 f+1}{4}, p\right)=\left|\frac{2 f+1}{4}-p\right| \leqslant|f-p|
$$

for all $f \in\left(\frac{1}{2}, 1\right]$. Finally, we generate the sequence (2.1) and show that it converges strongly to a fixed point of $T$. Choose $f_{1}=1 \in D=[0,1]$. Then $P_{\rho}^{\top}\left(f_{1}\right)=\frac{2 f_{1}+1}{4}=\frac{2(1)+1}{4}=\left\{\frac{1}{2}+\frac{1}{4}\right\}$ and $u_{1} \in P_{\rho}^{\top}\left(f_{1}\right)=$ $\left\{\frac{1}{2}+\frac{1}{4}\right\}$. That is, $u_{1}=\frac{1}{2}+\frac{1}{4}$. Let $h_{1}=(1-\gamma) f_{1}+\gamma u_{1}$. Then

$$
\mathrm{h}_{1}=\frac{1}{2}+\frac{1}{2}\left(\frac{1}{2}+\frac{1}{4}\right)=\frac{1}{2}+\frac{3}{8}, \quad \mathrm{P}_{\rho}^{\top}\left(\mathrm{h}_{1}\right)=\left\{\frac{2 \mathrm{~h}_{1}+1}{4}\right\}=\left\{\frac{2\left(\frac{1}{2}+\frac{3}{8}\right)+1}{4}\right\}=\left\{\frac{1}{2}+\frac{3}{16}\right\} .
$$

Choose $w_{1} \in P_{\rho}^{\top}\left(h_{1}\right)=\left\{\frac{1}{2}+\frac{3}{16}\right\}$, that is, $w_{1}=\frac{1}{2}+\frac{3}{16}$. Let $g_{1}=(1-\beta) u_{1}+\beta w_{1}$. Then

$$
g_{1}=\frac{1}{2}\left(\frac{1}{2}+\frac{1}{4}\right)+\frac{1}{2}\left(\frac{1}{2}+\frac{3}{16}\right)=\frac{1}{2}+\frac{7}{32}, \quad P_{\rho}^{\top}\left(g_{1}\right)=\left\{\frac{2 g_{1}+1}{4}\right\}=\left\{\frac{2\left(\frac{1}{2}+\frac{7}{32}\right)+1}{4}\right\}=\left\{\frac{1}{2}+\frac{7}{64}\right\} \text {. }
$$


Choose $v_{1} \in \mathrm{P}_{\rho}^{\top}\left(g_{1}\right)=\left\{\frac{1}{2}+\frac{7}{64}\right\}, v_{1}=\frac{1}{2}+\frac{7}{64}$. Let $f_{2}=(1-\alpha) v_{1}+\alpha w_{1}$. Then

$$
\begin{gathered}
f_{2}=\frac{1}{2}\left(\frac{1}{2}+\frac{7}{64}\right)+\frac{1}{2}\left(\frac{1}{2}+\frac{3}{16}\right)=\frac{1}{2}+\frac{19}{128}<\frac{1}{2}+\frac{1}{4}, \\
P_{\rho}^{\top}\left(f_{2}\right)=\left\{\frac{2 f_{2}+1}{4}\right\}=\left\{\frac{2\left(\frac{1}{2}+\frac{19}{128}\right)+1}{4}\right\}=\left\{\frac{1}{2}+\frac{19}{256}\right\} .
\end{gathered}
$$

Now choose $u_{2} \in P_{\rho}^{\top}\left(f_{2}\right)=\left\{\frac{1}{2}+\frac{19}{256}\right\}$, that is, $u_{2}=\frac{1}{2}+\frac{19}{256}$. Let $h_{2}=(1-\gamma) f_{2}+\gamma u_{2}$. Then

$$
\begin{aligned}
\mathrm{h}_{2} & =\frac{1}{2}\left(\frac{1}{2}+\frac{19}{128}\right)+\frac{1}{2}\left(\frac{1}{2}+\frac{19}{256}\right)=\frac{1}{2}+\frac{57}{512} \\
\mathrm{P}_{\rho}^{\top}\left(\mathrm{h}_{2}\right) & =\left\{\frac{2 \mathrm{~h}_{2}+1}{4}\right\}=\left\{\frac{2\left(\frac{1}{2}+\frac{57}{512}\right)+1}{4}\right\}=\left\{\frac{1}{2}+\frac{57}{1024}\right\} .
\end{aligned}
$$

Choose $w_{2} \in P_{\rho}^{\top}\left(h_{2}\right)=\left\{\frac{1}{2}+\frac{57}{1024}\right\}$, that is, $w_{2}=\frac{1}{2}+\frac{57}{1024}$. Let $g_{2}=(1-\beta) u_{2}+\beta w_{2}$. Then

$$
\begin{gathered}
g_{2}=\frac{1}{2}\left(\frac{1}{2}+\frac{19}{256}\right)+\frac{1}{2}\left(\frac{1}{2}+\frac{57}{1024}\right)=\frac{1}{2}+\frac{133}{2048} \\
P_{\rho}^{\top}\left(g_{2}\right)=\left\{\frac{2 g_{2}+1}{4}\right\}=\left\{\frac{2\left(\frac{1}{2}+\frac{133}{2048}\right)+1}{4}\right\}=\left\{\frac{1}{2}+\frac{133}{4096}\right\} .
\end{gathered}
$$

Choose $v_{2} \in \mathrm{P}_{\rho}^{\top}\left(\mathrm{g}_{2}\right)=\left\{\frac{1}{2}+\frac{133}{4096}\right\}, v_{2}=\frac{1}{2}+\frac{133}{4096}$. Let $\mathrm{f}_{3}=(1-\alpha) v_{2}+\alpha w_{2}$. Then

$$
f_{3}=\frac{1}{2}\left(\frac{1}{2}+\frac{133}{4096}\right)+\frac{1}{2}\left(\frac{1}{2}+\frac{57}{1024}\right)=\frac{1}{2}+\frac{361}{8192}<\frac{1}{2}+\frac{1}{6} .
$$

Continuing in a similar way, $\mathrm{f}_{4}<\frac{1}{2}+\frac{1}{8}, \mathrm{f}_{5}<\frac{1}{2}+\frac{1}{10}, \ldots, \mathrm{f}_{\mathrm{n}}<\frac{1}{2}+\frac{1}{n}$. This shows that $\left\{\mathrm{f}_{\mathrm{n}}\right\}$ converges strongly to a point of $F_{\rho}(T)=\left[0, \frac{1}{2}\right]$.

In the following example, we verify our Theorem 2.3.

Example 2.7. Consider the modular space $L_{\rho}=\mathbb{R}$ equipped with the norm $\|\cdot\|$, that is, $\rho(f)=|f|$ and $\mathrm{D}=\left\{\mathrm{f} \in \mathrm{L}_{\rho}: 1 \leqslant f<\infty\right\}$. Obviously $\mathrm{D}$ is a nonempty $\rho$-closed and $\rho$-convex subset of $\mathrm{L}_{\rho}=\mathbb{R}$. Define $\mathrm{T}: \mathrm{D} \rightarrow \mathrm{P}_{\rho}(\mathrm{D})$ by

$$
\mathrm{Tf}=\left[1,1+\frac{f}{2}\right]
$$

Then $\mathrm{F}_{\rho}(\mathrm{T})=[1,2]$. Let $\alpha=\beta=\gamma=\frac{1}{2}$.

Define a continuous nondecreasing function $l:[0, \infty) \rightarrow[0, \infty)$ by $l(r)=\frac{r}{4}$. Note that $\operatorname{dist}_{\rho}(f, T f) \geqslant$ $l\left(\operatorname{dist}_{\rho}\left(f, F_{\rho}(T)\right)\right)$ for all $f \in D$ as follows.

If $f \in F_{\rho}(T)=[1,2]$, then obviously

$$
\operatorname{dist}_{\rho}(f, T f)=0=l\left(\operatorname{dist}_{\rho}\left(f, F_{\rho}(T)\right)\right) .
$$

If $f \in(2, \infty)$, then we have

$$
\operatorname{dist}_{\rho}(f, T f)=\operatorname{dist}_{\rho}\left(f,\left[1,1+\frac{f}{2}\right]\right)=\left|f-\left(1+\frac{f}{2}\right)\right|=\frac{f-2}{2},
$$

and

$$
l\left(\operatorname{dist}_{\rho}\left(f, F_{\rho}(T)\right)\right)=l\left(\operatorname{dist}_{\rho}(f,[1,2])\right)=l(|f-2|)=\frac{f-2}{4} .
$$

So $\operatorname{dist}_{\rho}(f, T f) \geqslant l\left(\operatorname{dist}_{\rho}\left(f, F_{\rho}(T)\right)\right)$ for all $f \in D$ and hence the condition (I) is satisfied. Next note that 
$P_{\rho}^{\top}(f)=\{f\}$ when $f \in[1,2]$. If $f \in(2, \infty)$, then

$$
\begin{aligned}
P_{\rho}^{T}(f) & =\left\{g \in \operatorname{Tf}: \rho(f-g)=\operatorname{dist}_{\rho}(f, T f)\right\} \\
& =\operatorname{dist}_{\rho}\left(f,\left[1,1+\frac{f}{2}\right]\right) \\
& =\inf \left\{\rho(f-h): h \in\left[1,1+\frac{f}{2}\right]\right\} \\
& =\left\{g \in \operatorname{Tf}:|g-f|=\left|f-\left(1+\frac{f}{2}\right)\right|=\left|\frac{f}{2}-1\right|\right\} \\
& =\left\{g \in \operatorname{Tf}:|g-f|=\frac{f}{2}-1\right\} \\
& =\left\{g=1+\frac{f}{2}\right\} \text { because } f>g \text { for all } g \in \operatorname{Tf} \text { where } f \in(2, \infty)
\end{aligned}
$$

Now $\mathrm{P}_{\rho}^{\top}$ is $\rho$-quasi-nonexpansive because

$$
H_{\rho}\left(P_{\rho}^{\top}(f), P_{\rho}^{\top}(p)\right)=H_{\rho}\left(\left\{1+\frac{f}{2}\right\},\{p\}\right)=\left|1+\frac{f}{2}-p\right| \leqslant|f-p|, \text { as } f>2 .
$$

To generate the sequence $\left\{f_{n}\right\}$ of Theorem 2.3, we proceed as follows.

Choose $f_{1}=3 \in D=[1, \infty), P_{\rho}^{\top}\left(f_{1}\right)=1+\frac{3}{2}=\left\{\frac{5}{2}\right\}$ and $u_{1} \in P_{\rho}^{\top}\left(f_{1}\right)=\left\{\frac{5}{2}\right\}$. That is, $u_{1}=\frac{5}{2}$. Let $h_{1}=(1-\gamma) f_{1}+\gamma u_{1}$. Then

$$
\mathrm{h}_{1}=\frac{1}{2}(3)+\frac{1}{2}\left(\frac{5}{2}\right)=\frac{11}{4}, \quad \mathrm{P}_{\rho}^{\top}\left(\mathrm{h}_{1}\right)=1+\frac{\mathrm{h}_{1}}{2}=1+\frac{\frac{11}{4}}{2}=\left\{\frac{19}{8}\right\} .
$$

Choose $w_{1} \in P_{\rho}^{\top}\left(h_{1}\right)=\left\{\frac{19}{8}\right\}$, that is, $w_{1}=\frac{19}{8}$. Let $g_{1}=(1-\beta) u_{1}+\beta w_{1}$. Then

$$
g_{1}=\frac{1}{2}\left(\frac{5}{2}\right)+\frac{1}{2}\left(\frac{19}{8}\right)=\frac{39}{16}, \quad P_{\rho}^{\top}\left(g_{1}\right)=1+\frac{g_{1}}{2}=1+\frac{\frac{39}{16}}{2}=\left\{\frac{71}{32}\right\} .
$$

Choose $v_{1} \in \mathrm{P}_{\rho}^{\top}\left(\mathrm{g}_{1}\right)=\left\{\frac{71}{32}\right\}$, that is, $v_{1}=\frac{71}{32}$. Let $\mathrm{f}_{2}=(1-\alpha) v_{1}+\alpha w_{1}$. Then

$$
f_{2}=\frac{1}{2}\left(\frac{71}{32}\right)+\frac{1}{2}\left(\frac{19}{8}\right)=\frac{147}{64}=2+\frac{19}{64}<2+\frac{1}{2}, \quad P_{\rho}^{\top}\left(f_{2}\right)=1+\frac{f_{2}}{2}=1+\frac{\frac{147}{64}}{2}=\frac{275}{128}=\left\{\frac{275}{128}\right\} .
$$

Choose $u_{2} \in P_{\rho}^{\top}\left(f_{2}\right)=\left\{\frac{275}{128}\right\}$, that is, $u_{2}=\frac{275}{128}$. Let $h_{2}=(1-\gamma) f_{2}+\gamma u_{2}$. Then

$$
h_{2}=\frac{1}{2}\left(\frac{147}{64}\right)+\frac{1}{2}\left(\frac{275}{128}\right)=\frac{569}{256}, \quad P_{\rho}^{\top}\left(h_{2}\right)=1+\frac{\frac{569}{256}}{2}=\left\{\frac{1081}{512}\right\} .
$$

Choose $w_{2} \in P_{\rho}^{\top}\left(h_{1}\right)=\left\{\frac{1081}{512}\right\}$, that is, $w_{2}=\frac{1081}{512}$. Let $g_{2}=(1-\beta) u_{2}+\beta w_{2}$. Then

$$
\mathrm{g}_{2}=\frac{1}{2}\left(\frac{275}{128}\right)+\frac{1}{2}\left(\frac{1081}{512}\right)=\frac{2181}{1024}, \quad \mathrm{P}_{\rho}^{\mathrm{T}}\left(\mathrm{g}_{2}\right)=1+\frac{\mathrm{g}_{2}}{2}=1+\frac{\frac{2181}{1024}}{2}=\left\{\frac{4229}{2048}\right\} .
$$

Choose $v_{2} \in \mathrm{P}_{\rho}^{\top}\left(g_{2}\right)=\left\{\frac{4229}{2048}\right\}$, that is, $v_{2}=\frac{4229}{2048}$. Let $f_{3}=(1-\alpha) v_{2}+\alpha w_{2}$. Then

$$
f_{3}=\frac{1}{2}\left(\frac{4229}{2048}\right)+\frac{1}{2}\left(\frac{1081}{512}\right)=\frac{8553}{4096}=2+\frac{361}{4096}<2+\frac{1}{3} \text {. }
$$

In a similar way,

$$
\mathrm{f}_{4}=2+\frac{6859}{262144}<2+\frac{1}{4}
$$




$$
\begin{aligned}
f_{5}=2+\frac{130221}{16777216} & <2+\frac{1}{5}, \\
& \vdots \\
f_{n} & <2+\frac{1}{n} .
\end{aligned}
$$

This gives that $\lim _{n \rightarrow \infty} f_{n}=2 \in F_{\rho}(T)=[1,2]$. Hence we have the result.

\section{Acknowledgment}

The authors are grateful to the anonymous reviewers for their invaluable suggestions. We are thankful to Professor Ekrem Sevas for his cooperation in this work.

\section{References}

[1] M. Abbas, T. Nazir, A new faster iteration process applied to constrained minimization and feasibility problems, Mat. Vesnik, 66 (2014), 223-234. 1

[2] T. D. Benavides, M. A. Khamsi, S. Samadi, Asymptotically regular mappings in modular function spaces, Sci. Math. Jpn., 53 (2001), 295-304. 1

[3] B. A. B. Dehaish, W. M. Kozlowski, Fixed point iteration processes for asymptotic pointwise nonexpansive mappings in modular function spaces, Fixed Point Theory Appl., 2012 (2012), 23 pages. 1

[4] S. Dhompongsa, T. D. Benavides, A. Kaewcharoen, B. Panyanak, Fixed point theorems for multivalued mappings in modular function spaces, Sci. Math. Jpn., 63 (2006), 161-169.1

[5] M. A. Khamsi, W. M. Kozolowski, Fixed Point Theory in Modular Function Spaces, Springer, Berlin, (2015). 1, 1, 1, 1

[6] S. H. Khan, M. Abbas, Approximating fixed points of multivalued $\rho$-nonexpansive mappings in modular function spaces, Fixed Point Theory Appl., 2014 (2014), 9 pages. 1, 1, 1

[7] S. H. Khan, M. Abbas, S. Ali, Fixed points of multivalued quasi-nonexpansive mappings using a faster iterative process, Acta Math. Sin. (Engl. Ser.), 30 (2014), 1231-1241. 1, 2

[8] A. Razani, V. Rakocevic, Z. Goodarzi, Non-self mappings in modular spaces and common fixed point theorems, Cent. Eur. J. Math., 8 (2010), 357-366. 2.4, 2.5 